\title{
EQUIDAD Y JUSTICIA EN SALUD IMPLICACIONES PARA LA BIOÉTICA
}

\section{Marcela Ferrer Lues*}

Resumen: Lograr la equidad en salud es un desafío ético a nivel mundial. Si bien se sabe que la salud depende de diversos factores, la bioética se ha centrado en la equidad en los servicios de salud.

El conocimiento de los macrodeterminantes de la salud y sus efectos en las prácticas y análisis, como también los principios de autonomía y justicia, legitiman a la equidad en salud como un área sustantiva de la bioética. Algunos temas son: relación entre macrodeterminantes de la salud y estilos de vida; distribución social de la libertad de elección; tensiones éticas de las políticas de promoción de la salud; justicia en los servicios de salud; apreciación ética de la construcción de indicadores y su evaluación; definición de "salud" y "equidad en salud". El análisis de la equidad en salud abre nuevos campos de reflexión y práctica para una bioética centrada en la salud de la población.

Palabras clave: Bioética y equidad en salud, bioética y justicia social, bioética y salud pública, desigualdades en salud

\section{EQUITY AND JUSTICE IN HEALTH IMPLICATIONS FOR BIOETHICS}

\begin{abstract}
Achieving equity in health is an ethical challenge worldwide. Although it is known that health depends on diverse factors, bioethics has been focused on equity in health care.

The knowledge of the macro-determinants of health and their effects in the practices and analysis, as well as the principles of autonomy and justice, legitimize equity in health as a substantive area of bioethics. Some relevant issues are: relation between macro-determinants of health and lifestyles; social distribution of freedom of choice; ethical tensions in health promotion policies; justice in health care; ethical appreciation of construction of indicators and their evaluation; definition of "health" and "equity in health". The analysis of equity in health opens new fields of reflection and practice towards a bioethics centered in population health.
\end{abstract}

Key words: Bioethics and equity in health, bioethics and social justice, bioethics and public health, inequalities in health

\section{EQUIDADE E JUSTIÇA EM SAÚDE IMPLICAÇÕES PARA A BIOÉTICA}

Resumo: Alcançar a equidade em saúde é um desafio ético universal. Considerando-se que a saúde depende de diversos fatores, a Bioética tem se centrado na busca de equidade nos serviços de saúde.

O conhecimento dos macro-determinantes de saúde e seus efeitos práticos e analíticos, assim como os princípios da autonomia e justiça legitimam a equidade em saúde como área essencial de atenção da Bioética. Alguns temas de interesse são: relação entre macro-determinantes de saúde e estilos de vida: distribuição social da liberdade de escolha; tensões éticas relativas às políticas de promoção de saúde; justiça nos serviços de saúde; apreciação ética na elaboração de indicadores e suas avaliações; definição de "saúde" e "equidade em saúde". A análise de equidade em saúde abre novos campos de reflexão e prática para uma Bioética centrada na saúde da população.

Palabras chave: Bioética e equidade em saúde, bioética e justiça social, bioética e saúde pública, desigualdades em saúde

Socióloga. MHSc en Bioética. Profesora del Instituto de Asuntos Públicos e Investigadora del Centro Interdisciplinario de Estudios en Bioética, Universidad de Chile.

Correspondencia: ferrerm@chi.ops-oms.org 


\section{Introducción}

Lograr la equidad en salud es uno de los más importantes desafíos éticos a nivel mundial. Sin embargo, la bioética ha dedicado escasa atención a estos tópicos. El interés se ha centrado en la equidad en el acceso a los servicios de atención de salud, pese a que se ha demostrado que la salud depende de una diversidad de factores, entre ellos, los servicios de atención de salud. En este documento pretendo mostrar la relevancia que el tema de equidad en salud tiene para la bioética, y presentar algunas de las discusiones e interrogantes pendientes en esta área.

El artículo está dividido en dos partes. En la primera, discuto la necesidad y pertinencia que tiene para la bioética abordar el tema de las inequidades en salud como un área de trabajo sustantiva. Para ello me refiero a algunas circunstancias del desarrollo histórico y conceptual de la disciplina, las que explicarían en gran medida el desinterés por los temas de justicia en salud. Luego, analizo estas mismas circunstancias como fundamentos para la incorporación de tales temas, considerando tanto los avances en el conocimiento sobre los determinantes de la salud, como el rol de los principios de autonomía y justicia. En la segunda parte, presento algunas de las que podríamos llamar "certezas" y algunas de las preguntas pendientes en los análisis actuales sobre justicia ${ }^{1}$ en salud. Para ello, discuto la preponderancia de los macrodeterminantes de la salud por sobre los estilos de vida; las tensiones éticas de las políticas de promoción de "estilos de vida saludables"; la inexistencia de consenso respecto de las desigualdades injustas en salud -o lo que se conoce como "inequidades" en salud-; las alternativas de acción en el plano de las políticas públicas, y la

El término "equidad" se refiere a la "justicia". Su utilización responde a la necesidad de marcar una diferencia con el de "igualdad". En este documento utilizo el término "equidad" para referirme a las discusiones conceptuales respecto del término y "justicia” para aludir a la temática donde se sitúa esta discusión. ambigüedad que rodea lo que entendemos por "salud". Lejos de mis pretensiones está dar cuenta cabal de las discusiones actuales sobre estos temas. Me interesa más bien mostrar su pertinencia para la bioética, identificar algunas de las preguntas relevantes y motivar el desarrollo de una línea de trabajo que pareciera estar comenzando a cobrar fuerza al interior de la disciplina.

\section{Equidad en salud, justicia y bioética El desarrollo de la bioética y su definición temática}

Desde hace varios años, pero especialmente durante la última década, se ha demostrado que existen importantes diferencias en el estado de salud de las personas según distintos atributos socioeconómicos, sociodemográficos, geográficos, étnicos o de género. La difusión sistemática de estas diferencias ha permitido que el logro de la equidad en salud (valoración ética de estas diferencias) se transformara en uno de los principales objetivos de las agendas de los gobiernos y de los organismos internacionales. Pese al consenso en torno a la importancia de la equidad en salud, la bioética ha dedicado escasa atención a estas temáticas.

El escaso interés que la bioética ha mostrado por el tema de la equidad en salud o, más concretamente, el escaso interés demostrado por las personas que trabajan en ella, puede atribuirse a diversas razones, muchas de ellas ligadas al nacimiento de la bioética como disciplina ${ }^{2}$.

Los antecedentes relativos al nacimiento e historia de la bioética que expongo en este artículo se basan en la experiencia de los países desarrollados, principalmente de Estados Unidos. Hay quienes podrían afirmar que la bioética en América Latina tiene características distintivas y que, por tanto, este análisis no es aplicable a la Región. No incluyo una referencia específica a América Latina por dos razones. Primero, hasta donde tengo información, el análisis histórico del surgimiento y desarrollo de la bioética en esta Región sigue siendo una tarea pendiente. Segundo, la influencia de la bioética de los países desarrollados en América Latina, especialmente de la bioética norteamericana, es incuestionable. Después de todo, la bioética nace precisamente para dar respuesta a problemas que surgieron primero en los países desarrollados. 
Como señalan Callahan y Jennings(1), la bioética surgió a fines de la década de los sesenta y principios de los setenta, principalmente estimulada por los abusos sufridos por participantes en investigaciones, los movimientos emergentes de derechos de los pacientes y los dilemas éticos asociados a los avances tecnológicos aplicados en medicina. Más que interesarse en la salud de la población, la bioética se centró en el bienestar del individuo, en particular en su autonomía. Típicos temas de salud pública, tales como las desigualdades sociales y económicas, recibieron escasa atención.

El posterior desarrollo de la bioética consolidó también el desinterés por la relación entre desigualdades socioeconómicas y salud. Al respecto, Wikler(2) distingue cuatro fases de desarrollo. La primera fase consistió en la discusión de códigos de conducta profesional, donde la bioética era más bien "ética médica". La segunda fase correspondió al nacimiento de la bioética como tal, que incluyó la participación de médicos y no médicos en la discusión y elaboración de nuevas formas de la relación médico-paciente. El punto de inicio de la tercera fase podría ubicarse en 1980, con un llamado de Daniel Callahan ${ }^{3}$ a mirar la estructura, financiamiento y organización de los servicios de salud, puesto que la relación médicopaciente y todas las preocupaciones de la segunda fase estaban controladas en gran medida por ellos. Responder a este desafío era difícil. Se necesitaba una formación en economía de la salud y filosofía política, particularmente en teorías de justicia distributiva, materias todas escasamente conocidas por quienes trabajaban en bioética. Los trabajos de Norman

El autor cita la Shattuck Lecture como la primera vez que Daniel Callahan aborda estos temas públicamente. La bioética europea también comienza a tratar los temas de justicia durante los años ochenta, principalmente a través del tema de SIDA como problema social.
Daniels $^{4}$ sobre justicia en los servicios de atención de salud son clave en este período.

La cuarta y última fase, en la cual nos encontraríamos en la actualidad, es definida por Wikler como Bioética de la salud de la población. Sus diferencias con las etapas anteriores serían: a) los dilemas relacionados con la aplicación de alta tecnología a la medicina pierden su centralidad. El foco de atención se ubica en los muchos determinantes de la salud, uno de los cuales es la medicina de alta tecnología; b) el foco de atención es tanto en la salud como en los servicios de atención de salud. No sólo interesa el acceso a los servicios por parte de quienes están enfermos, sino también los factores que explican que algunas personas se enfermen más que otras. Temas centrales son los relacionados con la medición de las condiciones de salud, los valores que guían las políticas públicas y los macrodeterminantes ${ }^{5}$ de la salud; c) se otorga una importancia al peso relativo de ciertos grupos sobre el total de la población para priorizar las decisiones y las áreas de trabajo a nivel nacional e internacional; d) se requiere un mayor sentido de las prioridades, poniendo mayor energía en las áreas que afectan a los grupos más desfavorecidos; e) se requiere un nuevo aparato conceptual. En síntesis, la bioética de la salud de la población pone atención en la salud y no sólo en los servicios de atención de salud; incluye a todos, independientemente de su status o bienestar, poniendo énfasis en los pobres; incluye todos los determinantes de la salud y tiene por conceptos clave los de equidad, igualdad y justicia, carga de

Para una exposición sintética y reciente de los planteamientos de Norman Daniels respecto de los macrodeterminantes de las condiciones de salud, la justicia en salud y la justicia en los servicios de atención de salud, ver Daniels N. Justice, Health and Healthcare. The American Journal of Bioethics Spring 2001; 1(2).

5 Los "macrodeterminantes de la salud" se refieren a variables socioeconómicas, sociodemográficas o culturales que afectan el estado de salud, tales como ingreso, etnicidad, género, área de residencia, educación, entre otras. 
enfermedad y costo-efectividad, y determinación de prioridades. Asumir esta cuarta fase es para Wikler una responsabilidad social y requiere que las personas que trabajan en el área se formen en campos hasta ahora no familiares, como son los de salud pública, salud internacional, análisis costo-efectividad y medición de la salud.

Sin duda la propuesta de Wikler incluye una lectura tanto de los avances ocurridos en la bioética como de las necesidades sociales que dieron origen a esos cambios. La bioética surge para "humanizar" la relación médico-paciente y orientar la toma de decisiones frente a los desafíos del avance tecnológico. Posteriormente, la comprensión de que la relación médicopaciente depende en gran parte de la estructura de los servicios de atención de salud, legitimó el interés de la bioética en el tema de la justicia en el acceso a estos servicios. El reconocimiento de que el acceso a los servicios de atención de salud es sólo un factor que incide en las condiciones de salud, una de las mayores contribuciones de los estudios de desigualdades en salud, permite ahora legitimar el trabajo de la bioética en el tema de la equidad en salud o, como Wikler llama, la bioética de la salud de la población.

La identificación de la salud como un fenómeno multidimensional, que no depende exclusiva ni mayoritariamente del acceso a los servicios de atención de salud, ha permitido importantes avances en el campo de los análisis y las acciones en el ámbito de la salud, entre ellos la legitimación del trabajo interdisciplinario; la desmitificación de la tecnología como la panacea para la resolución de todos los males; la valoración de las disciplinas distintas de la medicina en su contribución a la salud; el reconocimiento de la necesidad de abordar las "interrelaciones" de los distintos sectores o componentes de lo que podría entenderse como "condiciones de vida"; el análisis de las relaciones -más bien confusas- entre "calidad de vida" y "salud". Esto no es ajeno a la revalorización de la democracia como sistema político, al ejercicio de la ciudadanía como derecho de las personas y a la participación social como necesaria para el desarrollo. Tampoco es ajeno al reconocimiento del desarrollo como distinto del crecimiento económico; al reconocimiento de la necesidad de respetar a las minorías y a las identidades nacionales; a la valoración de la equidad de género. Aunque muchos de estos "reconocimientos" se dan más a nivel de discurso que en la práctica concreta, no es casual que estos aspectos sean también incluidos en los análisis de desigualdad en salud. Su inclusión respondería tanto a los avances en el conocimiento como a las transformaciones sociales y políticas de los últimos tiempos, y a sus sinergias.

El reconocimiento de la multicausalidad de la salud legitima incluir en la bioética no sólo el análisis de la equidad en salud, sino también otros temas, tales como el de medio ambiente y biodiversidad, el de las relaciones entre bioética y salud pública, o el de calidad de vida, temas que parecieran estar cobrando cada vez mayor interés ${ }^{6}$. En este marco, el discurso bioético ha reordenado los temas tradicionales generando nuevas síntesis, las cuales se centran en dilemas humanos y no en rígidas fronteras de especialidades(3). La incorporación de estos temas implica una creciente legitimación del trabajo interdisciplinario en bioética y un mayor reconocimiento del aporte de otras disciplinas distintas de la medicina, en particular de las ciencias sociales. Al mismo tiempo, sugiere un cuestionamiento de la definición de la bioética como disciplina, en tanto el trabajo interdisciplinario -o si se quiere, transdiscipli-

El VI Congreso Mundial de Bioética realizado en Brasilia, a principios de noviembre del 2002, mostró un número importante de trabajos en estas temáticas. 
nario- implica una combinación -o fusión- de las perspectivas de distintas disciplinas frente a un área de trabajo común, en este caso la salud. Los alcances de esta discusión y sus impactos sobre la bioética como disciplina son profundos y escapan los objetivos de este trabajo. Probablemente la discusión de estos temas cobrará mayor importancia en los próximos años.

\section{Los principios de la bioética y su definición temática}

Más allá de las demandas actuales de la bioética y de la incorporación de nuevas temáticas de trabajo, el escaso interés en los temas de equidad y justicia en salud que han mostrado quienes trabajan en bioética podría verse asociado no sólo a sus orígenes históricos, sino también a sus bases conceptuales. Evidentemente esto no es casual, ya que el aparato conceptual de cualquier disciplina está en relación con sus ámbitos de acción y su historia, definiendo a la vez estos mismos ámbitos. El énfasis en los cuatro principios de la bioética ${ }^{7}$ explicaría, desde mi perspectiva, gran parte del desinterés por los temas de justicia y equidad en salud.

Los principios de autonomía, beneficencia, no maleficencia y justicia definen comportamientos esperados, derechos y deberes para personas que desempeñan roles específicos, de acuerdo con su relación con los profesionales

\footnotetext{
El Informe Belmont, elaborado en 1979 por la Comisión de Protección de los Sujetos de la Investigación Biomédica y Conductual de Estados Unidos, identificó los principios de respeto por las personas, beneficencia y justicia. Estos principios fueron posteriormente definidos por Beauchamp y Childress como autonomía, beneficencia, no maleficencia y justicia. Ver: The National Commission for the Protection of Human Subjects of Biomedical and Behavioral Research. The Belmont Report: Ethical Principles and Guidelines for the Conduct of Research Involving Human Subjects. Washington D.C.: Department of Health, Education, and Welfare; 1979. También: Beauchamp T, Childress J. Principios de Ética Biomédica. Barcelona: Masson S.A.;1999.
}

de la salud o los investigadores. Precisamente, por esa identificación como pacientes o participantes en una investigación, ellos son claramente diferentes de las personas que son definidas como beneficiarias de las políticas públicas. Estos beneficiarios potenciales no sólo son anónimos, sino, además, carecen de poder para hacerse reconocer y autorreconocerse, ya que muchos de ellos viven en la pobreza, son discapacitados, pertenecen a minorías étnicas o poseen otras similares condiciones de exclusión.

La bioética ha mostrado especial énfasis en el principio de autonomía y en los derechos individuales. Este énfasis se manifiesta principalmente en la excesiva preponderancia otorgada al consentimiento informado, lo que en la práctica es, en muchos casos, un mero requisito para evitar problemas legales. Los principios de no maleficencia y beneficencia tienen también una base individual, ya sea para aprobar tratamientos médicos, evaluar protocolos de investigación o definir situaciones en las cuales las personas son incapaces de otorgar su consentimiento.

Por su parte, el principio de justicia se refiere también a individuos concretos y establece que cada uno de ellos debe ser tratado en forma justa. Pese a tener esta base individual, incluye también una clara referencia a otros pacientes/participantes, sus grupos de pertenencia y a la sociedad en su conjunto. Esto porque determinar la justicia de una distribución requiere, necesariamente, considerar las posiciones relativas de los miembros de la sociedad con respecto al objeto o bien específico que se quiere distribuir.

En concordancia con el principio de justicia, la bioética ha realizado importantes contribuciones en el ámbito de la justicia en el acceso a los servicios de atención de salud y en la asignación 
de recursos ${ }^{8}$. Esto puede ser entendido como una forma de afectar positivamente a la salud, ya que el mejoramiento de los servicios de atención de salud debiera contribuir a mejorar las condiciones de salud. Sin embargo, existe una amplia literatura que muestra que el acceso a los servicios de atención de salud es sólo uno de los factores que determina la salud de la población. Desigualdades en salud han sido reportadas en países como Canadá y el Reino Unido(4), ambos parte del mundo desarrollado, con políticas universales de acceso a los servicios de atención de salud. Desigualdades en salud en América Latina en función de diversas variables socioeconómicas también han sido reportadas $(5,6)$.

El principio bioético de justicia requiere poner mayor atención a los macrodeterminantes de salud y no sólo a los servicios de atención de salud, en la medida en que el logro de la equidad -o justicia- en salud requiere de acciones orientadas a esos determinantes. Esto no está solamente relacionado con el principio de justicia, sino también con el principio de autonomía. Como discuto en la siguiente sección, los estilos de vida han sido identificados como uno de los factores determinantes de las condiciones de salud. Las diferencias en salud causadas por estilos de vida libremente elegidos serían justas, en tanto las restricciones a la libertad de elección generarían desigualdades injustas -las denominadas "inequidades en salud"-. Estos temas abren una importante discusión para la bioética, centrada principalmente en la autonomía, la libertad en la elección de estilos de vida y los condicionamientos sociales y económicos de la autonomía. Los principios de justicia y autonomía no son rivales sino complementarios porque, asegurar tanto el respeto a la autonomía como las condiciones para su ejercicio pleno, constituye también un asunto de justicia.

Ver, por ejemplo, Daniels N, Sabin J. Setting Limits Fairly. Can We Learn to Share Medical Resources? New York: Oxford University Press; 2002.

\section{Justicia en salud: ¿qué diferencias son justas?}

\section{Macrodeterminantes de la salud y estilos de vida}

Existe una consistente evidencia empírica que demuestra la existencia de desigualdades en salud. Por lo general, estas desigualdades se entienden como significativas diferencias en las condiciones de salud (paradojalmente medidas por esperanza de vida al nacer, tasas de mortalidad infantil, mortalidad por causas o tasas de morbilidad) entre diferentes grupos socioeconómicos, definidos de acuerdo con su nivel de ingreso, educación, origen étnico, género, área de residencia $u$ otros factores similares.

Aunque existe acuerdo en que el acceso a los servicios de atención de salud es sólo uno de los factores que influyen en la salud de la personas, existe desacuerdo respecto de qué tipo de factores tiene mayor influencia. Existen dos posiciones principales: quienes identifican a las condiciones socioeconómicas y quienes identifican a los estilos de vida o decisiones individuales(7). La primera explicación se relaciona con el principio de justicia, mientras que la segunda con el principio de autonomía.

En la medida en que algunos factores que predisponen a ciertas enfermedades se han vuelto más conocidos y fáciles de controlar o evitar, los estilos de vida han sido identificados como importantes determinantes de la salud. Por esta vía, las personas están siendo crecientemente rotuladas como responsables por enfermarse. Sin embargo, la responsabilidad que una persona tiene respecto de su enfermedad es cuestionable, ya que la enfermedad puede ser resultado tanto de elecciones individuales como de las condiciones de injusticia de la sociedad. Más aún, la libertad de elección es desigualmente distribuida o los resultados de un estilo de vida no son verdaderamente elegidos(8). Por ejemplo, podemos vivir en una subcultura donde fumar juega un papel importante en el establecimiento de relaciones socia- 
les, tal como ocurre en muchos grupos de adolescentes. Los miembros de ese grupo "eligen" fumar, pero su libertad de elección está claramente constreñida por la pertenencia a ese grupo. Segundo, en condiciones de libertad de elección los fumadores no eligen enfermarse de cáncer al pulmón; eligen fumar y tomar el riesgo de contraer cáncer al pulmón.

Además de los condicionantes socioeconómicos a la libertad de elección y al hecho de que una elección libre implica más que nada elegir un riesgo, los estilos de vida sólo explican una parte de las condiciones de salud o enfermedad. Por ejemplo, algunos estudios han encontrado diferencias en la mortalidad por cáncer al pulmón según distintos estratos socioeconómicos o niveles educacionales, las cuales no son explicadas por el hábito de fu$\operatorname{mar}(9,10)$. Esto indicaría que la reducción de la incidencia del cáncer de pulmón debiera ser buscada no sólo por campañas orientadas a disminuir el hábito de fumar, sino también mediante políticas orientadas a disminuir las desigualdades económicas y sociales.

Para nadie debiera ser novedad que las posibilidades reales de modificar patrones de comportamiento ligados a la salud, tales como tener una dieta sana, no fumar, beber alcohol con moderación o realizar actividades físicas, están más cercanas a los estratos socioeconómicos medios y altos ${ }^{9}$. Lo mismo es válido para trabajar en ambientes saludables, alternar jornadas de trabajo con jornadas de descanso, rea-

La Carta de Ottawa para la Promoción de la Salud, producto de la Primera Conferencia Internacional sobre Promoción de la Salud, realizada en 1986, identificó algunos requisitos previos para la salud como son la paz, un ecosistema estable, justicia social y equidad, y recursos como educación, alimentos e ingresos. Esto implica reconocer las conexiones entre las condiciones sociales y económicas, los cambios estructurales, el entorno físico, los modos de vida individual y la salud. Ver: Quinta Conferencia Mundial de Promoción de la Salud: Hacia una mayor equidad. Ciudad de México, 5-9 de junio del 2000. [Sitio en Internet]. Disponible en http://www.paho.org/ Spanish/HPP/mexdec2000.pdf Acceso en junio de 2003. lizar actividades recreativas y otras actividades similares. El énfasis en la promoción de "estilos de vida saludables" por sobre la modificación de las desigualdades sociales y económicas podría incluso contribuir a mantener o aumentar la brecha entre los distintos estratos socioeconómicos(11), brecha que también ha sido identificada como un factor contribuyente a las desigualdades en salud(12).

Aun cuando existieran las condiciones para una libre elección de estilos de vida "saludables", los estilos de vida no tienen más que un efecto probabilístico en la salud. Este efecto probabilístico, reconocido o no explícitamente por las campañas de promoción de la salud, hace referencia a importantes preguntas éticas ligadas a la relación individuo-Estado. Entre ellas, las relacionadas con el rol del paternalismo en las políticas públicas o la legitimación de la acción del Estado en "protegernos de nosotros mismos(8)". Estas preguntas corresponden a típicas preocupaciones de la bioética: el grado en que las políticas de salud pueden oponerse al principio de autonomía.

Las tensiones individuo-Estado, o bienestar individual-bienestar social, abren una diversidad de temas para la bioética. Entre ellos, la responsabilidad de las personas respecto de sus estilos de vida; el rol del gobierno en crear las condiciones para que las personas puedan ejercer esa responsabilidad; el rol del gobierno en impedir o promover comportamientos relacionados con la salud o en desarrollar programas educativos; el uso de incentivos para promover la salud, y la importancia relativa para la sociedad de buscar una buena salud(1). Estas preguntas se relacionan no sólo con la tensión individuo-Estado, sino también con las posibilidades de "libertad de elección" que existen en una sociedad. En la medida en que existe una desigual distribución de la libertad de elección, el análisis debe incorporar necesariamente 
las bases de esta desigual distribución, que responden a las condiciones de justicia o injusticia presentes en la sociedad.

\section{Inequidades y desigualdades en salud}

Existe consenso a nivel mundial en que las desigualdades en salud deben ser disminuidas. Sin embargo, el logro de la equidad en salud, y no el de la igualdad en salud, es frecuentemente mencionado. En otras palabras, existe acuerdo en aceptar cierto grado de desigualdad en salud, pero existe desacuerdo en torno a qué grado de desigualdad es moralmente aceptable. En la medida en que el desacuerdo en la definición de la justicia -o más precisamente de lo que se conoce como "equidad" en salud- persiste, los estudios empíricos sólo pueden medir "desigualdades en salud" y no "inequidades en salud".

Debido a sus implicaciones éticas, definir las inequidades en salud es una tarea extremadamente difícil, existiendo una mínima posibilidad, si alguna, de llegar a un acuerdo respecto de una definición universal. Describir una situación como "equitativa" requiere identificar lo moralmente adecuado de ciertas distribuciones, ya sean desiguales o iguales. Esto implica que la justicia se refiere a la igualdad, pero no exclusivamente a ella. La igualdad respondería a otras propiedades de relevancia moral: los iguales debieran recibir igual tratamiento, mientras que los desiguales debieran recibir un tratamiento desigual, en proporción a las desigualdades moralmente relevantes. Esto corresponde a las nociones de igualdad horizontal y vertical aristotélicas, que inspiraron los trabajos de Sen sobre justicia y desigualdad $^{10}$. El reconocimiento de la diversidad y de las diferencias humanas ha llevado a

Almeida-Filho N. Theories of equity and justice in a globalized world (and how they relate to the problem of health inequality). Notas para la sesión plenaria del VI Congreso Mundial de Bioética. Brasilia: octubre de 2002. plantear la necesidad de una sociedad "equitativa" o, en otras palabras, una sociedad desigualmente justa.

Los conceptos de equidad horizontal y vertical tienen importantes implicancias para las políticas públicas, pues se traducen en una asignación diferencial de recursos. Tomando como criterio de asignación la necesidad, esto significa entregar recursos equivalentes para iguales niveles de necesidad y recursos diferentes para distintos niveles de necesidad. La equidad vertical tiene un mayor poder redistributivo $\mathrm{y}$ por eso muchas veces encuentra mayores obstáculos. Sin embargo, quienes piensan limitar la acción pública y expandir la acción del mercado, han valorado la equidad vertical como mecanismo para satisfacer las necesidades de quienes tienen una privación severa de recursos financieros. La interpretación de quienes tienen estas privaciones suele ser estrecha, dejando a amplios sectores en condiciones de gran necesidad(13).

La definición frecuentemente mencionada de equidad en salud fue la elaborada por Margareth Whitehead a principios de los años 90(14). De acuerdo con esta definición, el concepto de equidad en salud tiene una dimensión moral y ética. Refiere a diferencias que no sólo son innecesarias y evitables, sino también injustas. La definición de injusticia requiere considerar el contexto, es decir, lo que se considera justo o injusto en una determinada sociedad.

Si bien Whitehead reconoce que el análisis de la equidad en salud requiere considerar el contexto donde se producen las desigualdades, plantea que existe consenso en la literatura en que las desigualdades en salud, originadas en diferencias biológicas y en comportamientos libremente elegidos, no son injustas y, por tanto, no corresponden a inequidades en salud. En otras palabras, las desigualdades en salud originadas en diferencias biológicas parecieran ser 
inevitables y las originadas en comportamientos libremente elegidos parecieran ser justas. Sin embargo, las desigualdades en salud que resultan de condiciones socioeconómicas y ambientales externas al individuo, incluidos los estilos de vida, serían consideradas por muchos como injustas, ya que la mayoría de estas condiciones serían evitables.

No cabe duda de que el trabajo de Whitehead ha significado un importante avance para el análisis de las desigualdades e inequidades en salud. Los estudios teóricos y empíricos realizados en los últimos diez años, como también las iniciativas para la formulación de políticas públicas, coinciden con esta definición y tienden a definir a las inequidades en salud como todas aquellas diferencias en las condiciones de salud que se originan en condiciones socioeconómicas, culturales o medioambientales que son innecesarias, evitables e injustas. No obstante este consenso en la definición, aún no existe consenso en definir qué desigualdades en salud son inequidades o, en otras palabras, en operacionalizar esta definición. Las dificultades se relacionan con la identificación de lo que es evitable, innecesario e injusto y las relaciones entre estas condiciones(15). La más aceptada definición de "equidad en salud" es, paradojalmente, incapaz de proveer los criterios para determinar precisamente las desigualdades en salud que pueden ser consideradas justas, lo que se refleja en una reiterada inconsistencia conceptual.

La definición de las desigualdades justas e injustas en salud es un desafío teórico de plena vigencia. Las posibilidades de avance son mínimas o nulas si lo que se busca es contar con una definición de justicia o equidad en salud que pueda ser aplicable a distintas sociedades y que permita comparar inequidades en salud entre países -y no desigualdades-. Para esto se requiere formular una teoría de justicia distributiva de consenso universal, algo evidentemente poco probable. Lo mismo es válido incluso a nivel nacional, porque se requiere el consenso de los diferentes grupos y corrientes de pensamiento que componen esa sociedad particular. ¿Debemos entonces olvidarnos del tema y pensar en aquellos ámbitos en los que sí podemos lograr consenso ${ }^{11}$ ? Aunque ésta pareciera ser la mejor solución en el plano de las políticas públicas, está lejos de ser una solución en el plano de las ideas y del desarrollo teórico. Renunciar a la posibilidad de contar con una definición universal de equidad en salud; reconocer que mucho de lo que se compara y muestra corresponde a "desigualdades" y no a "inequidades"; incorporar el conocimiento que entregan los estudios empíricos para orientar el diseño de políticas públicas; refinar los procedimientos de registro de información para su monitoreo, y admitir que lo que se busca es una sociedad "desigualmente" justa son, sin duda, algunos de los criterios orientadores de la acción pública. Reflexionar sobre qué significa justicia en salud, cuáles son las fuentes de esa injusticia, cómo contribuye la justicia social a la justicia en salud y qué relaciones existen entre salud y desarrollo son, sin duda, las preguntas claves desde el mundo de las ideas y el desarrollo teórico.

\section{Equidad en salud y políticas públicas}

Si bien las reflexiones teóricas orientan la acción pública, sus ámbitos de competencia son esencialmente distintos. Desde la perspectiva de las políticas públicas no se trata de conceptualizar la equidad, aunque el contenido de las políticas responde de una u otra forma a una aproximación teórica. Se trata funda-

\footnotetext{
11 Esa es precisamente la propuesta de Daniels y Sabin en su teoría de justicia en los servicios de atención de salud, que se centra en la justicia del proceso y no de los resultados. Ver: Daniels N, Sabin J. Setting Limits Fairly. Can We Learn to Share Medical Resources? New York: Oxford University Press; 2002.
} 
mentalmente de demostrar que una situación es inequitativa, en orden a asignar recursos para disminuir esas disparidades. Criterios recurrentes mencionados en la literatura para identificar distribuciones injustas son: evitabilidad, elección y agente responsable(13). Una situación inequitativa sería aquella en que las diferencias en la distribución de un bien, por ejemplo de los recursos de salud o de los macrodeterminantes de las condiciones de salud, son financiera, técnica y moralmente evitables, no reflejan libre elección, y las demandas debieran identificar un agente responsable, ya sea como originador o como agente con capacidades para rectificar la desigual distribución.

Evidentemente, estos criterios son útiles para orientar el proceso de formulación de demandas en el plano de políticas públicas. Sin embargo, su puesta en práctica aún requiere una teoría de justicia para definir, desde el punto de vista moral, la evitabilidad de las diferencias y las posibilidades de libertad de elección. La fundamentación técnica no es ajena a estos juicios morales(2), en tanto la medición de las desigualdades lleva implícita una evaluación ética que guía la construcción y utilización de los indicadores, orientando no sólo la determinación de qué aspectos de la realidad son incluidos, sino también qué magnitud es considerada como relevante en materia de desigualdad. Todos estos temas implican un campo de acción relevante para la bioética y remiten tanto al desarrollo de una teoría de justicia en salud(15), como a la formulación de orientaciones de políticas públicas dirigidas a disminuir las inequidades.

La ausencia de consenso sobre cuáles desigualdades en salud son injustas no implica la ausencia de orientaciones para la formulación de políticas públicas. Como dice Gwatkin(16), los distintos análisis de las desigualdades en salud -que él identifica como orientados a la pobreza, a la igualdad o a la equidad-, tendrían diferencias más bien académicas que de orientación práctica. Después de todo, señala el autor, quienes buscan la igualdad o la equidad no plantean disminuir las diferencias mediante la disminución de los niveles de salud de los miembros de los estratos socioeconómicos más favorecidos, sino a través del mejoramiento de las condiciones de los grupos menos favorecidos. El énfasis en la pobreza implica la necesidad de redefinir los objetivos de salud planteándolos no en términos de indicadores promedio nacionales o regionales, sino en términos de los grupos menos favorecidos de la sociedad. Esto implica la necesidad de generar información y formas de medición para el diseño y evaluación de políticas públicas de salud, tareas todas que implican una evaluación ética de los instrumentos y los resultados.

Si bien es cierto que focalizar las acciones en la población pobre tendría como resultado probable una disminución de las desigualdades de salud entre los pobres y los ricos, este enfoque enfrenta, desde mi perspectiva, al menos dos dificultades. Primero - y frecuentemente mencionado en los análisis sobre focalización de las políticas públicas-, focalizar en la población pobre implica excluir a la población que se encuentra inmediatamente después del límite de pobreza definido. Esta población, que se encuentra en condiciones relativas ligeramente mejores, puede empeorar sus condiciones producto de la falta de atención; el resultado puede ser tan sólo un intercambio de población entre los pobres y quienes se encuentran cercanos al límite de la pobreza. Segundo, la evidencia disponible señala que las desigualdades en salud se manifiestan en la forma de una gradiente que implica un mejoramiento de las condiciones de salud a medida que se asciende en la escala socioeconómica. Precisamente por esta gradiente, disminuir las desigualdades requiere ir más allá de las acciones orientadas a 
disminuir la pobreza(17). Esta evidencia señala, incluso, que la magnitud del ingreso individual sería tan importante como la distribución del ingreso a nivel de la sociedad: sociedades con una mayor desigualdad en el ingreso tendrían una mayor desigualdad en salud, independientemente de los niveles de ingreso de esa sociedad en su conjunto(12). En este marco, la búsqueda de una teoría de justicia en salud o equidad en salud, o la identificación de las desigualdades en salud que son evitables, innecesarias o injustas, tiene plena vigencia. Igualmente vigente es la discusión en torno al concepto de salud, en tanto define qué es aquello que esperamos sea distribuido de manera justa o equitativa.

\section{Equidad en salud: ¿qué es salud?}

Diez años atrás, Margareth Whitehead definió "equidad en salud". Aunque esta definición no ha estado libre de críticas, pareciera ser la mejor disponible. Cincuenta años atrás, la Organización Mundial de la Salud (OMS) definió el concepto de "salud". Esta definición pareciera ser también la mejor disponible, pero ha sido frecuentemente criticada. Esto significa que sabemos cuáles son las fuentes que causan desigualdades injustas en salud (con un rango de evaluación que depende de criterios éti$\cos$ ), pero tenemos una ambigua definición de lo que consideramos salud. De hecho, los indicadores comunes utilizados para mostrar desigualdades - que en algunos casos son erróneamente definidos como "inequidades"- en condiciones o "resultados" de salud, son más bien condiciones o "resultados" de enfermedad: esperanza de vida, tasas de mortalidad, mortalidad por causas, morbilidad y otros.

Como ha sido frecuentemente mencionado, la OMS define salud como un completo estado de bienestar físico, mental y social y no solamente ausencia de enfermedad. Esta definición tiene el mérito de incorporar el bienestar social y de ampliar la salud más allá de sus aspectos físicos y mentales. Esto sería un antecedente para desarrollar conceptos orientados a entregar un enfoque más comprehensivo en salud, como sería el de calidad de vida ${ }^{12}$. Pese a estos aspectos positivos, la definición de la OMS no provee un marco satisfactorio para definir las condiciones de salud y es demasiado amplia para guiar la formulación de políticas públicas.

Desde la perspectiva de las políticas públicas, la definición de la OMS tiene dos consecuencias distintas, pero simultáneas, para las políticas de salud y las prácticas de atención de salud. Por un lado, otorga un significado más comprehensivo de salud. Mejorar las condiciones de salud de la población es un objetivo que debiera ser seguido por distintos sectores sociales -al menos educación, empleo, vivienda, medio ambiente y salud-como también por los profesionales de la salud, profesionales de otras disciplinas, pacientes y la población en general. Esta visión comprehensiva de la salud estimula el desarrollo de enfoques interdisciplinarios y la formulación de políticas intersectoriales.

Por otro lado, entender la salud como multidimensional e intersectorial implica una ambigüedad tanto respecto de sus límites como de quien es responsable de proveerla. Posiblemente esta ambigüedad explica por qué hablar de salud refiere generalmente a enfermedad: los servicios de atención de salud atienden principalmente a la población enferma; las desigualdades en salud son presentadas como diferencias en morbilidad o mortalidad en función de determinadas condiciones socioeconómicas; reducir las inequidades en salud en los países en desarrollo significa disminuir la incidencia de las enfermedades infecciosas. La mayoría

12 Para una discusión sobre las dificultades de homologar el concepto de salud con el de calidad de vida, ver: Samaja J. Las condiciones de vida y la salud. PAHO Report. HDP/ HST. 1994. 
de las llamadas "prácticas de atención de salud" o "políticas de salud" continúan centrándose en la enfermedad y en la población enferma, como también los profesionales de la atención de salud son frecuentemente identificados como los únicos profesionales que deben hacerse cargo de la salud. Evidentemente esta identificación, que frecuentemente es también una autoidentificación, tiene impactos negativos cuando no se refiere solamente a la atención médica destinada a atender, sanar o acompañar la enfermedad, sino también a otras actividades relacionadas, como son las de administración de los servicios de salud, la investigación en salud o las relacionadas con las políticas de salud.

Las dificultades para definir el concepto de salud han sido discutidas por muchos autores ${ }^{13}$. Sin embargo, la filosofía no ha desarrollado enfoques alternativos y se ha centrado principalmente en el concepto de enfermedad(18). Ni las ciencias sociales ni las ciencias de la salud han desarrollado un concepto alternativo de salud que pueda ser aceptado por la comunidad científica en su conjunto. A las dificultades en la definición de la equidad en salud ya descritas se suman las dificultades en la propia definición de salud. Definir "equidad en salud" como distinto de "equidad en enfermedad" es un desafío pendiente. Asumir este desafío es indispensable para la formulación de políticas públicas orientadas precisamente a aumentar las condiciones de equidad en salud.

Más allá de la importancia de definir "salud" como requisito para el diseño de políticas públicas, la definición de salud es, en sí misma, un asunto relativo a la justicia. La definición de salud como multidimensional, el énfasis en los macrodeterminantes de la salud y el papel cada vez menos importante que se le ha asignado a los servicios de atención de salud

Ibíd. en las condiciones de salud de la población, ha permitido importantes avances pero, a la vez, ha generado cierta confusión. El énfasis en la influencia que las condiciones sociales y económicas tienen en las condiciones de salud, en detrimento de los servicios de atención de salud, tiene como conclusión lógica que tanto más justa es una sociedad, más saludable es su población. Las intervenciones públicas orientadas a mejorar la distribución de los macrodeterminantes de la salud se convierten en condiciones necesarias para mejorar la salud de la población. Sin embargo, este argumento puede ser también utilizado para disminuir o negar la obligación del Estado de asegurar el acceso de las personas a los servicios de atención de salud: si los servicios de atención de salud tienen un escaso rol en la salud de la población ¿por qué el Estado debiera proveerlos? Este argumento iría acompañado de propuestas que otorgan al "libre mercado" la tarea de distribuir "equitativamente" los macrodeterminantes de la salud. Esta posición es contraria a todos quienes creemos que el Estado tiene la obligación moral de asegurar la atención de salud de la población, en tanto es condición indispensable para la vida humana. El cumplimiento de esta obligación no es más que una expresión del respeto a la dignidad humana, de la justicia y del grado de solidaridad presentes en una determinada sociedad.

El reconocimiento de la importancia de los macrodeterminantes de la salud no implica olvidarnos de los servicios de atención de salud, ya que aún cuando tengamos una justa distribución de los macrodeterminantes de la salud, la población seguirá enfermándose y necesitando atención médica(15-17). Esto es mayormente válido para las personas que llegan a edades más avanzadas, lo que tiene una mayor importancia a nivel social no sólo por el aumento en la esperanza de vida al nacer y en especial a los sesenta años, sino también por el 
fenómeno de compresión de la morbilidad en esas edades. El análisis de las condiciones de justicia en las condiciones de salud y en el acceso a los servicios de atención de salud, de la justicia en la distribución de los recursos de salud y el establecimiento de prioridades, de las relaciones entre justicia social y justicia en salud, y de las relaciones entre salud y desarro1lo, así como también las necesarias definiciones de "equidad", "salud" y "equidad en salud" son sin duda temas indispensables de abordar.

\section{Conclusiones}

Una de las mayores contribuciones de los estudios sobre desigualdades en salud ha sido identificar la salud como un fenómeno multidimensional, que no depende exclusiva ni mayoritariamente del acceso a los servicios de atención de salud. Esto ha permitido importantes avances en el campo de los análisis y las acciones en el ámbito de la salud. No sólo por estos avances, sino también en virtud de los principios de autonomía y justicia, la bioética debiera integrar los temas de equidad y justicia en salud como una de sus áreas sustantivas.

La preponderancia de los macrodeterminantes de la salud por sobre los estilos de vida, la desigual distribución de la libertad de elección, las tensiones éticas de las políticas de promoción de "estilos de vida saludables", la discusión sobre cuáles son las desigualdades injustas en salud - $\mathrm{O}$ "inequidades" en salud-, la justicia en los servicios de atención de salud, las alternativas de acción en el campo de las políticas públicas, la apreciación ética de la construcción de indicadores y su evaluación, y la definición de qué entendemos por "salud", abren una variedad de temas que permiten incorporar el análisis de la equidad en salud a la bioética o, desde una perspectiva más amplia y tal como define Wikler, desarrollar una bioética de la salud de la población.

\section{Referencias}

1. Callahan D, Jennings B. Ethics and Public Health: Forging a Strong Relationship. American Journal of Public Health 2002; 92 (2): 169-176.

2. Wikler D. Presidential Address: Bioethics and Social Responsibility. Bioethics 1997; 11 (3-4): 185192.

3. Lolas F. Bioética. Santiago de Chile: Editorial Universitaria; 1998: 61.

4. London Health Observatory. Report mapping out health inequalities across the city. [Sitio en Internet]. Disponible en $\backslash t$ «_blank» http://www.lho.org.uk/pubs/p_pubs.htm Acceso en junio de 2003.

5. Casas JA, Dachs JNW, Bambas A. Health Disparities in Latin America and the Caribbean: the Role of Social and Economic Determinants. En: Equity and Health: Views from the Pan American Sanitary Bureau. Washington DC: Pan American Health Organization. [Sitio en Internet]. Disponible en: http:/ /www.paho.org/English/DBI/Op08/OP08_03.pdf Acceso en junio de 2003.

6. OPS. Revista Panamericana de Salud Pública Mayo-Junio 2002. Número especial sobre factores determinantes de la inequidad en salud; 11 (5-6).

7. Peter F. Health Equity and Social Justice. Journal of Applied Philosophy 2001; 18 (2). 
Equidad y justicia en salud. Implicaciones para la bioética - M. Ferrer

8. King's Fund. Public Health and Public Values 2001. [Sitio en Internet]. Disponible en http:// www.kingsfund.org.uk/ePublicHealth/assets/applets/public.pdf Acceso en noviembre de 2002.

9. Hart CL, Hole DJ, Gillis ChR, et al. Social class differences in lung cancer mortality: risk factor explanations using two Scottish cohort studies. International Journal of Epidemiology 2001; 30: 268274.

10. Martikainen P, Lahelma E, Ripatti S, et al. Educational differences in lung cancer mortality in male smokers. International Journal of Epidemiology 2001; 30: 264-267.

11. Leichter H. Lifestyle Correctness and the New Secular Morality. En: Brandt A, Rozin P. Morality and Health. New York: Ed. Routledge; 1997.

12. Kawachi I, Kennedy B, Wilkinson R, eds. The Society and Population Health Reader. Vol. 1. Income Inequality and Health. New York: The New Press New York; 1999.

13. Bambas L, Casas J.C. Assessing Equity in Health: Conceptual Criteria. En: Equity and Health: Views from the Pan American Sanitary Bureau. Washington D.C.: Pan American Health Organization 2001: 12-21. [Sitio en Internet]. Disponible en http://www.paho.org/English/DBI/Op08/OP08_02.pdf Acceso en junio de 2003.

14. Whitehead M. Los conceptos y principios de la equidad en la salud. Organización Panamericana de la Salud 1991. Washington D.C.: Centro de Documentación e Información. Programa de Desarrollo de Políticas de Salud. Serie Reprints; 9.

15. Daniels N. Why justice is good for our health. En : Lolas F, Agar L, eds. Interfaces between bioethics and the empirical social sciences. Santiago de Chile: Programa Regional de Bioética OPS/OMS; 2002: $37-52$.

16. Gwatkin DR. Health inequalities and the health of the poor: What do we know? What can we do? Bulletin of the World Health Organization 2000; 78 (1) Ref. No. 0287.

17. Daniels N, Kennedy B, Kawachi I. Justice is good for our health. How greater economic equality would promote public health. In: Cohen J, Roger J, eds. Is Inequality Bad for Our Health? Boston: Beacon Press; 2000.

18. Engelhardt T, Wildes K. Health and Disease: Philosophical Perspectives. En Reich Warren T, ed. Encyclopedia of Bioethics 1995: 1101-1106. 